\title{
Electron Tomography Workflows using Scipion
}

Jorge Jiménez ${ }^{1}$, Federico De Isidro ${ }^{1}$, Estrella Fernández Jiménez ${ }^{2}$, David Herreros Calero ${ }^{2}$, Yunior Fonseca $^{1}$, Pablo Conesa ${ }^{1}$, Ana Cuervo ${ }^{1}$, Javier Conesa ${ }^{1}$, Roberto Melero ${ }^{1}$, Jose Maria Carazo ${ }^{1}$ and Carlos Oscar Sorzano 1

${ }^{1}$ CSIC, United States, ${ }^{2}$ CSIC, Madrid, Spain

Electron Tomography is an emerging imaging technique that allows the visualization of biological macromolecules in situ. A typical image processing pipeline includes aligning the movies (if acquired in this modality), alignment of the tilt series, CTF determination, 3D reconstruction with CTF correction, and the identification of the macromolecules of interest (subtomograms), subtomogram alignment, and classification. Different software packages address the different steps (Xmipp, MotionCorr2, CTFFind, NovaCTF, IMOD, Eman, Dynamo, Pyseg, Relion). We have integrated all these packages and some useful housekeeping tools within Scipion to become interoperable, and the whole workflow is traceable and reproducible. In this work, we present the tools available in Scipion for Electron Tomography.

Scipion is a workflow engine specially designed for the execution of image processing workflows in CryoElectron Microscopy. It is well-known in Single Particle Analysis [1], where it integrates more than 30 different packages and over 300 protocols. Scipion has been recently expanded to include atomic modeling of the reconstructed maps [2]. Now, we have extended Scipion to cover workflows in Electron Tomography. The included software goes through the whole image processing pipeline from the acquired movies to the electron tomogram, particle identification, and subtomogram averaging. One of the most interesting features of Scipion is the possibility of designing protocols that compare the output of multiple algorithms performing the same task so that consensus results can be calculated.

Scipion has its own data models for Electron Tomography and protocols calling the different packages convert the data to and from the desired package. In this way, one may perform the movie alignment with one package, the tilt series alignment with another, the 3D reconstruction of the tomogram with yet another, and all the operations required for the interoperation of the different packages are transparently performed by the engine. 


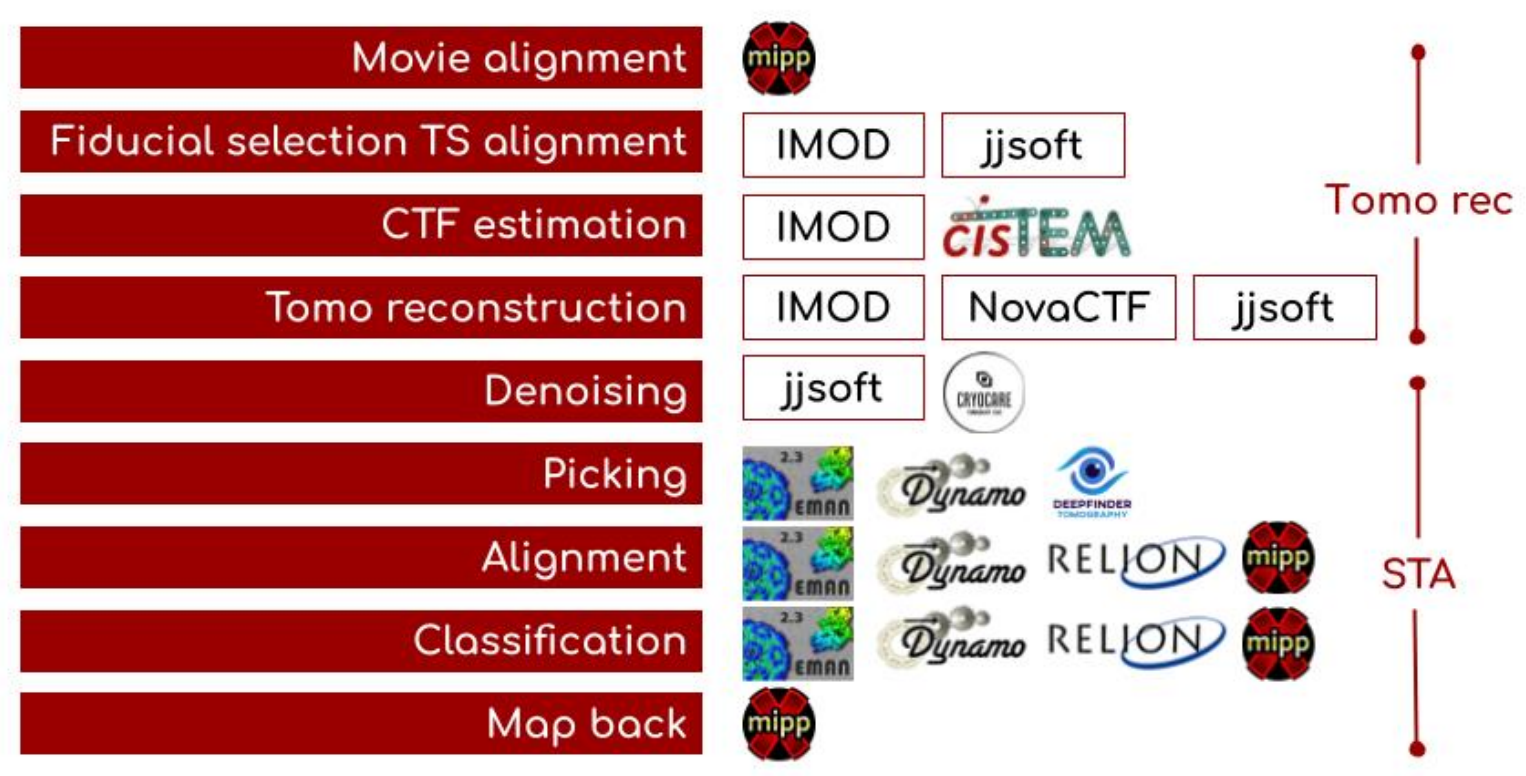

Figure 1. Image processing pipeline for Electron Tomography and integrated packages addressing these steps.

\section{References}

[1] J.M. de la Rosa-Trevin, A. Quintana, L. del Cano, A. Zaldivar-Peraza, I. Foche, J. Gutierrez, J. GomezBlanco, J. Burguet-Castells, J. Cuenca-Alba, V. Abrishami, J. Vargas, J. Oton, G. Sharov, J.L. Vilas, J. Navas, P. Conesa, M. Kazemi, R. Marabini, C.O.S. Sorzano, J.M. Carazo. Scipion: a software framework toward integration, reproducibility, and validation in 3D Electron Microscopy. J. Structural Biology, 195: 93-99 (2016)

[2] M. Martínez, A. Jiménez-Moreno, D. Maluenda, E. Ramírez-Aportela, R. Melero, A. Cuervo, P. Conesa, L. Del Caño, Y.C. Fonseca, R. Sánchez-García, D. Strelak, J.J. Conesa, E. Fernández-Giménez, F. de Isidro, C.O.S. Sorzano, J.M. Carazo, R. Marabini. Integration of Cryo-EM Model Building Software in Scipion. J. Chemical Information and Modelling 60: 2533-2540 (2020) 